interviewing, focused physical examination, and diagnostic assessment. At a weighty 640 pages, it is unlikely to find its way into many white coat pockets - an unfortunate fact given the clarity and utility of much of the information within.

The first and largest section of the book comprises 17 chapters intended to direct history taking and physical examination across all organ systems, including specific advice for pediatric and geriatric patients, and is a clear highlight of the text. The majority of the chapters employ a similar format. Relevant anatomy is briefly introduced through illustrations, followed by frequent chief complaints for a given system. The reader is then guided through a symptom-focused exam, describing information that should be elicited from the patient during the medical history and proper technique of pertinent physical exam maneuvers. The clinical examination is presented in a clear, logical manner that will mirror the flow of a successful doctor-patient interview. Each chapter concludes with a discussion of potential follow-up tests and imaging and their applicability to a number of common clinical presentations. Interspersed throughout the text are "clinical pearls," asides that range from must-not-miss constellations of symptoms to appropriate timelines for disease screening to simple admonitions ("Make sure fingernails are trimmed!," p. 126).

Less successful are the 10 "Essentials" chapters, which provide additional insights into clinical specialties, including endocrinology, emergency medicine, infectious disease, and oncology, as well as more general topics such as pharmacology, fluid repletion, and imaging modalities. Certain chapters (Essentials of Dermatology, notably) are useful guides to common symptoms that would not be out of place among earlier chapters, though the hand-drawn depictions of rashes will do little to inspire confidence in one's ability to identify them on a patient. Too often, however, the information in these chapters is presented in a dense, table-based manner akin to study guides for USMLE Step 1, making it nearly impossible to integrate into a successful patient interaction.
Finally, the three appendices to the text cover the basics of evidence-based practice and clinical epidemiology, frequently used drugs for a variety of clinical scenarios, and common laboratory values, respectively. Of these, the tables of lab results prove the most useful, providing normal ranges, critically elevated, or decreased levels, and notes on their significance in an easy to browse format.

While its lack of depth may render Essentials of Clinical Examination less useful in a specialty practice, medical, nursing, and physician assistant students should find the practical history and physical exam guidance invaluable. As an introductory reference to primary-care patient encounters, the book is highly recommended.

\section{Gregory Stachelek}

Department of Genetics

Yale School of Medicine, Class of 2016

Immunology: An Illustrated Outline. Fifth Edition. By David Male. New York: Garland Science, Taylor \& Francis Group; 2014. 147 p. US \$31.40 (Paperback). ISBN: 978-0815345015.

Although this book states that it was written with three functions in mind, it is actually a concise reference of basic immunology for a broad audience, including those currently reading immunology and those who have not previously studied immunology. The inclusion of both contents pages and an index of terms broaden its accessibility to a non-specialist audience and make it a convenient read for the specialist, thus making it incredibly clear and easy to navigate. The five topics are arranged logically and cover a comprehensive introduction to the basics of immunology, clinical immunology, and immunological techniques. The illustrations complement and further clarify the succinct text nicely; importantly, both color schematics and micrographs are included, which are very insightful. Furthermore, the inside cover contains a glossary of icons used throughout the book. This continuity really helps unify and simplify the information provided. This book is very successful at pro- 
viding the necessary information that enables the reader to glean a conceptual understanding of whatever aspect of immunology they choose to learn.

\author{
Natalie A. Roberts \\ Department of Molecular, Cellular, \\ and Developmental Biology \\ Yale University
}

\section{Clinical Application of Computer- Guided Implant Surgery. By Andreas Parashis and Panagiotis Diamantopou- los. Boca Raton, FL: CRC Press; 2014. 159 p. US \$179.95 (Hardcover). ISBN: 978-1482205411.}

This text will be a helpful resource for any practitioner of dental implant surgery. It is concise, heavily illustrated, and full of practical examples of computer-guided surgery. At times, the reader may find the captioning of figures to be sparse, but there is certainly no shortage of figures themselves.

The book is structured as two chapters of high-level description of the use of computerguided implant surgery, followed by a series of case studies, and closes with a multi-chapter summary of the method's benefits and efficacy.

In keeping with the style of the entire book, the first substantial chapter describes diagnosis and treatment planning for computerguided implant surgery primarily through figures. The crux of the advised treatment pathway is summarized in a single table, accompanied by recommendations for imaging techniques and a description of the function of available computer software. The next chapter covers guide selection, clearly explaining the surgical guide decision pathways using sparse flow charts and, again, plentiful photographs.

About a third of the text is devoted to case studies, documenting 13 real clinical singletooth, partial endentulism, and complete endentulism scenarios. Each case briefly describes the patient's circumstances, presents a bulleted treatment plan, and contains numerous photographs, medical radiographic images, and screen captures of software interfaces and computer renderings. These examples help to build a tangible understanding of the practice and utility of computer-guided implant surgery.

In the penultimate chapter of the text, we learn of the many advantages of computerguided implant surgeries - and also the relatively few drawbacks. The final chapter provides evidence of the accuracy of the method by citing the results of several published studies. There is also a brief presentation as to how drill diameter and length will affect surgical accuracy.

Clinical Application of Computer-Guided Implant Surgery provides high-level guidelines toward the application of computer-guided implant surgery and will perhaps be most valued for the comprehensive case studies.

\section{Mary Germino \\ Department of Biomedical Engineering Yale University}

\section{The Neurobiology of Learning and Memory. 2nd Edition. By Jerry W. Rudy. Sunderland, MA: Sinauer Associates, Inc.; 2014. 435 p. US \$78.16 (Hard- cover). ISBN: 978-1605352305.}

Rudy's text is well-suited for the student of neuroscience and should also find an audience among constituents of peripheral fields such as pyschology or biology. The reader will be served well to have some familiarity with the underlying biochemistry, but an ample review of foundational material is provided. It manages to capture the breadth of the subject without becoming too dense.

This text provides nearly a full chapter of historical context in the introduction, with additional anecdotal details peppered throughout the remainder of the book; these make for an enjoyable read. Each scientific theory is supported by published experimental findings and case studies. The book strives to remain impartial in the presentation of controversial theories, providing scientific evidence and suggesting interpretations without imposing definitive conclusions when not warranted. Influential experiments and their results are described in detail and copiously illustrated.

The topic of memory is covered comprehensively from a neurobiologic perspective, 